\title{
A Projection Color Auto-adjustment Technology in Landscape Design Teaching
}

\author{
http://dx.doi.org/10.3991/ijet.v11i09.6119 \\ Yue Wang \\ Sichuan University of Science and Engingeering, Zigong, China
}

\begin{abstract}
In the fast-paced modern life where high efficiency is pursued, projection technology is not only applicable to temporary conferences and workshops for technology and network center, but is also connected with the computer and multimedia. Thus, projection technology is a large-screen image equipment projection technology that is widely applied. An intelligent projection system with the function of background color auto-adjustment was constructed. In the present paper, the intelligent projection system was combined with multimedia teaching of Landscape Design. Application results show that the image shown on the multimedia large screen by the projection display system is clearer than common multimedia, and the color effect is more vivid. Thus, this projection display system improves the teaching quality of Landscape Design, including exhibition, appreciation, communication, and examination, and enhances the teaching effect and the students' learning ability.
\end{abstract}

Index Terms - landscape design, auto-adjustment, image projection, multimedia

\section{INTRODUCTION}

Projection technology is a kind of multimedia technology that utilizes light source decomposition and transformation and projection principle for display [1]. At present, the classification of projection technology is diversified, mainly including CRT technique [2], LED technique [3], DLP technique [4], etc. In modern society, the application scope of projection technology is extensive. For example, an audio-visual situation can be created with projection technology in such occasions of temporary conference, technology workshop, education and teaching, monitoring and commanding. In this way, users can clearly and vividly view and learn an audiovisual display [5].

The audio-visual display advantage of projection technology is evident. In recent years, projection technology has been increasingly introduced in teaching and education. Based on this premise, researches on the application of relevant projection technology in modern education and teaching are increasing. For example, Stone, J. A. et al. [6] integrated video images of action and ball projection technology in four different conditions: Integrated Video and Ball Projection (VBP), Video-Only (VO), Ball Projection-Only (BPO), and Misleading Ball Projection (MBP) and indicated that advance information and vision of a ball's trajectory prior to ball release play a significant role for successful performance and that integrated projection technology may provide a typical design for the research on interceptive actions. Shu and $\mathrm{Hu}$ [7] proposed that traditional mental experiment teaching mode brings considerable inconvenience and numerous defects for teaching, and that the application of computer and multimedia projection technology made up for such defects. Shu and $\mathrm{Hu}$ [8] verified through teaching experiment that multimedia projection technology can improve teaching quality and the students' learning interest. A research team established a multi-projective plane immersion teaching system, including hardware environment and software implementation. Furthermore, $\mathrm{Shu}$ and $\mathrm{Hu}[8]$ constructed a $2 * 2$ high-resolution display system with PC cluster and DLP projector to offer a learning environment with a large viewing angle and a sense of immediacy for learners and achieve immersion learning experience. Liu et al. [9] applied the microscopic projection system to the core course of medical examination technology specialty - Foundation of Clinical Examination. Results show that microscopic projection technology can fully mobilize the students' learning initiative and enthusiasm, enhance interactions between teachers and students, and effectively improve the teaching effect of experiment teaching.

The aforementioned scholars studied the application of projection technology in modern teaching from different perspectives and offered beneficial reference for generalizing and optimizing projection technology in teaching. However, existing projection technology also has some defects. Among these defects, the most notable one is that projection color cannot be auto-adjusted according to background color, which results in poor projection effect. In particular, when the wall surface is used for projection background, the color cast may easily occur. Thus, the present paper developed and applied a multimedia system that can auto-adjust projection color. Such system has a positive effect on improving projection color effect and projection color adaptivity.

\section{SYSTEM OVERVIEW}

\section{A. General design of system}

The projection color auto-adjustment multimedia system introduced in the present paper is a high-end multimedia projection device based on artificial intelligence technology [10] and information technology. The system consists of three parts: mobile device, projection equipment, and projection background. In the specific application, the mobile device obtains a color coefficient of the projection background through the internal camera, and the adaptive technique in artificial intelligence is applied to adopt this coefficient to the color that complies with projection background color and feed back the color to the projection equipment. The three parts form a color loop system to complete the projection display of the material object's color. In addition, the mobile device's camera is used to complete the 
optimization and adjustment of the projection background color without the camera. This step in the process improves the practical color's reliability and applicability. Based on the aforementioned design thought, the general design diagram of this system is drawn in Figure 1.

As shown in Figure 1, the system carries out adaptive adjustment of projection background color by introducing artificial intelligence technology. In the operation process, the first projection color parameter can be generated only by the mobile device through the interactions of mobile device, artificial intelligence system, and projection equipment. Thus, the camera on the projection equipment has to be installed. As a result, the projection color quality improves, and the production cost of the whole system also decreases.

\section{B. Key technology of system}

In this system's development and design, some core techniques are required to guarantee clear and fluent projection pictures and high color self-adaption, including geometric correction, chromatic aberration correction, artificial intelligence, and so on. The technical process of the whole system is shown in Figure 2.

As shown in Figure 2, artificial intelligence selfadaption, geometric correction, and chromatic aberration correction are key techniques in the system's technical process. First, the geometric correction technique is mainly used to solve the distortion of a single object projected on the screen, or a picture connection problem among multiple projectors. With the geometric correction technique, the whole projection picture can become more continuous. Second, chromatic aberration correction is used to solve chromatic aberration deviation of objects on the projector and provides observers with better visual effect. Artificial intelligence self-adaption carries out selfadaptive correction of projection background color through optimization algorithm on the basis of artificial intelligence technology so that the color of the object projected on the large screen can reflect the original color of the furthest object. Meanwhile, the color adapts to the screen's background color to achieve the optimal display effect of the object's projection color.

\section{System module composition}

Based on the aforementioned design thought, projection color adaptive adjustment multimedia system is divided into two parts and six modules in the specific design. The master-slave mode's design architecture is adopted to form the system information hardware layout. In the detailed work, each module focuses on the adaptive adjustment's core function. Figure 3 shows the module structure diagram of this system.

The system is mainly composed of two parts: projection subsystem and mobile subsystem. The projection subsystem is divided into receiving module (radio frequency receiving technology, used to receive projection collection signal transmitted by mobile subsystem), projection module (used to transmit the processed video signal to the projection background), CPU processing module (central processing module, used to handle chromatic aberration and geometric correction), and program operation triggering module (terminal module triggered by data computation). The mobile subsystem includes transmitting module (radio frequency receiving technology, used to transmit background color system signal to the projection module),

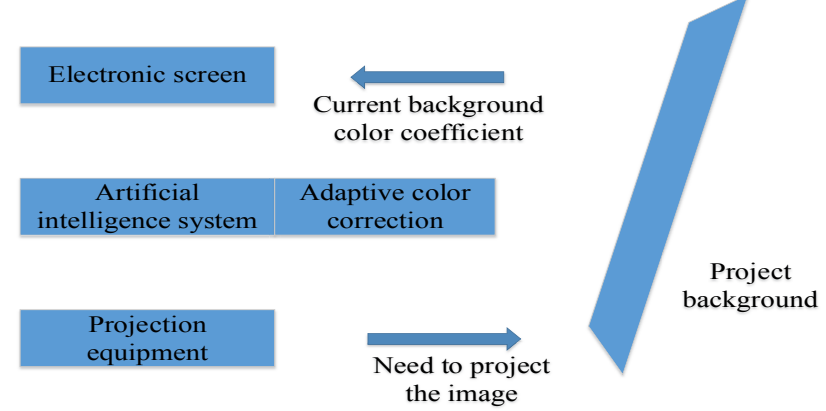

Figure 1. General design diagram of system

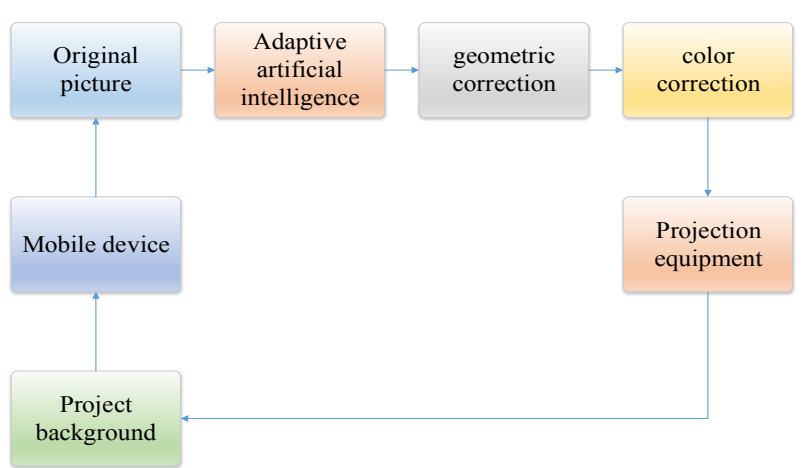

Figure 2. Technical process diagram of system

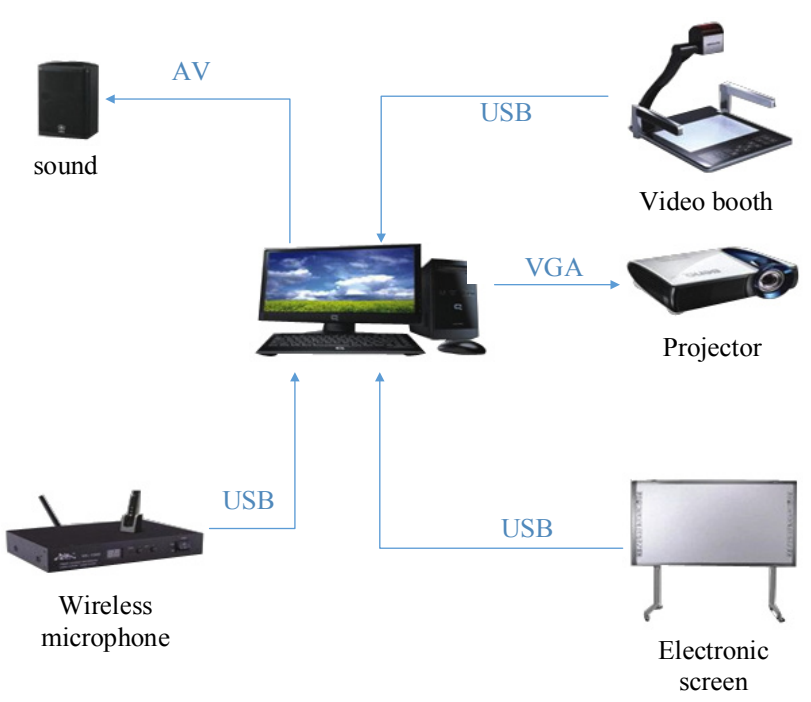

Figure 3. System module composition

artificial intelligence module (adaptive adjustment of color), storage module (used to store color image data), and wireless module (used to gather, process and transmit wireless signals).

\section{TEACHING EXPERIMENT ANALYSIS}

\section{A. Selection of teaching objects}

The students in Class (1) and Class (2) of 2013 landscape architecture specialty in the university were chosen as the objects of the study. Each class comprised 30 students, and there was no significant difference in the students' age, gender, and learning ability $(\mathrm{P}<0.05)$. In the specific teaching experiment, Class (1) served as the control class, whereas Class (2) served as the experimental class. 
PAPER

A Projection Color Auto-Adjustment TeChNOlogy in LANDSCAPE Design TeAching

\section{B. Teaching design}

In the specific teaching, Landscape Design of landscape architecture specialty was chosen as the main content, and the course involved 36 class hours. The course was composed of theory and practice teaching. To highlight the teaching value of the multimedia system with adaptive adjustment of image projection color, this system was used to organize teaching for the experimental class, whereas traditional multimedia equipment was used for the control class. After the course teaching, learning interest, examination score, and color cognition ability of students in both classes were gathered and analyzed, and the teaching effects were compared.

\section{Statistical method}

The SPSS13.0 software was applied to analyze the students' learning interest and color cognition ability. $\mathrm{P}<0.05$ means that the difference has statistical significance. In addition, Microsoft EXCEL 2010 software was used for the data statistics of examination scores, and a graph was made for the analysis.

\section{Teaching organization form}

\section{1) Organization form of control class}

In teaching the control class, the teacher combined traditional multimedia equipment with teaching method for teaching. The detailed organization form is as follows: PowerPoint (PPT) and blackboard were used as the teaching tools, and classroom explanation, question answering, assignment, and practical training were combined for teaching practice.

\section{2) Organization form of experimental class}

In teaching the experimental class, the teacher employed the projection color auto-adjustment multimedia system as the tool to organize teaching. "Landscape system of tourist area" in Landscape Design was taken as an example in the teaching. Under the support of the adaptive teaching system, the whole teaching organization includes the following steps:

First, case frame exhibition. Before the course started, the teacher used this system to display landscape design cases in some new tourist areas in China. There are more or less some defects in these cases. The teacher magnified these defects, constructed a sound design frame, and showed the sound design frame to students. Under the support of the color adaptive system, the key points of all defects were exhibited in a complete and careful manner. Students could immediately understand the teacher's teaching intention through observation, while the teacher mainly guided the students to discuss and explore in classroom, as shown in Figures 4 and 5.

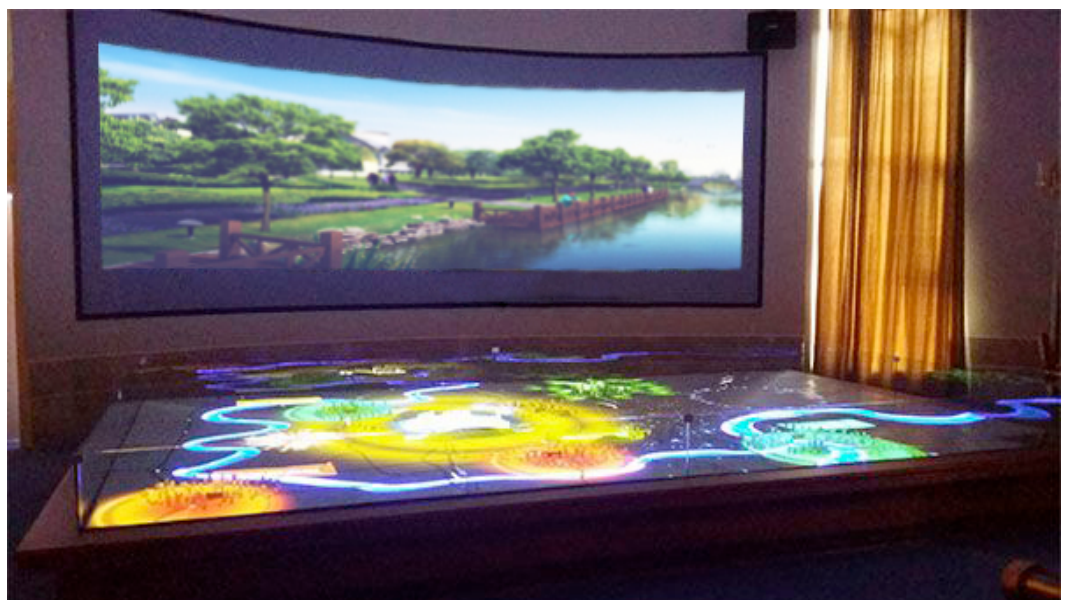

Figure 4. Teaching text material

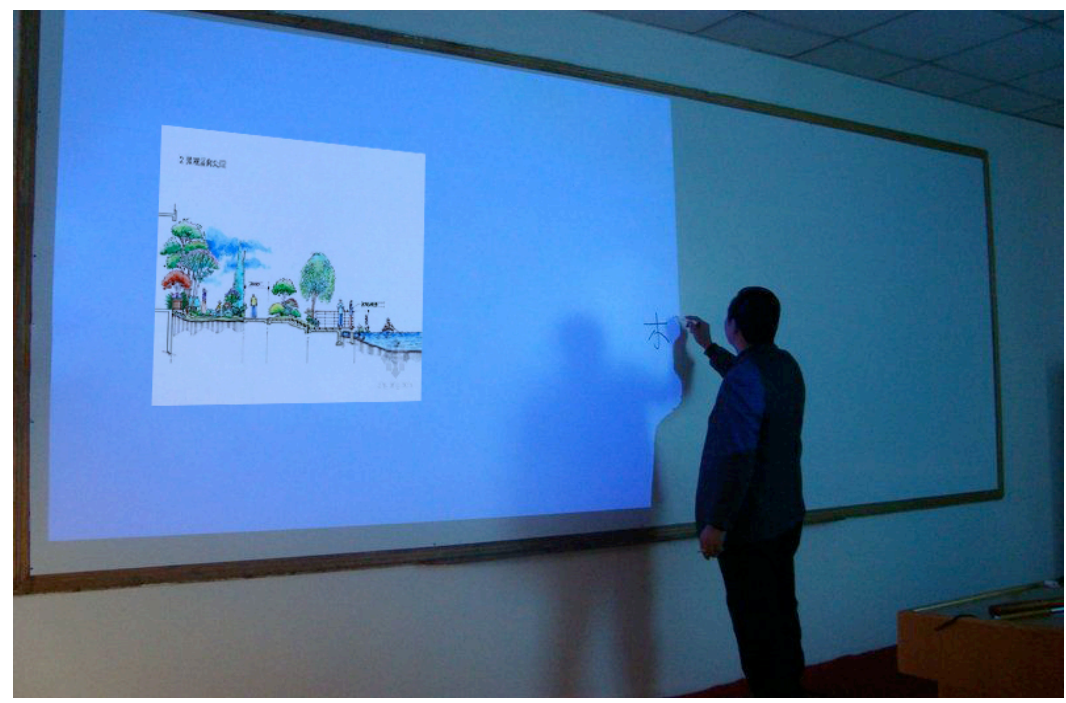

Figure 5. Teaching text material 
Second, classroom practice. After the observation in the initial stage, the teacher grouped the students in the class, and each group was given a specific design task to improve the defective landscape. The members of each group created a scheme to improve landscape design according to the observation, group exploration, and cooperation and formed the detailed text and picture design report. Thus, the students conducted classroom design practice.

Third, works exhibition. When all groups completed the design, the teacher held a seminar with the theme of landscape design spot demonstration and applied image projection color auto-adjustment technology in the multimedia. Each group selected a representative to upload the improved works to the memorizer through the mobile device and used the system as the exhibition carrier for classroom demonstration and reporting. Each group learned from each other and communicated on the seminar. The system set up a perfect color situation for teaching demonstration. Compared with traditional multimedia equipment, this system can better display the redesign of landscape in the tourist area, and the effect is great.

Fourth, examination and evaluation organization. After each teaching item was finished, the teacher organized an examination according to the aforementioned process, i.e., defective case exhibition-classroom design practiceworks demonstration. Compared with the traditional examination mode, the examination of each item can become more vivid, efficient, and interesting with the help of adaptive color adjustment system.

\section{TEACHING RESUlt AND Discussion}

After the 36-hour class, the teacher adopted the questionnaire survey, theoretical examination, and practical training examination for the statistics of learning indexes of experimental class and control class. The specific statistical data are as figure 6 .

Based on Figure 6, before the multimedia system with adaptive adjustment of image projection color is introduced, the leaning indexes of the experimental class and control class had no significant statistical difference. After the 36-hour class, the students in the experimental class were significantly superior to the students in the control class in terms of learning interest, color cognition ability, group cooperation ability, and independent inquiry ability, and the data comparison has significant statistical difference. Therefore, the introduction of the multimedia system with the adaptive adjustment of image projection color plays a significant role in improving the students' learning ability. (Figure 7)

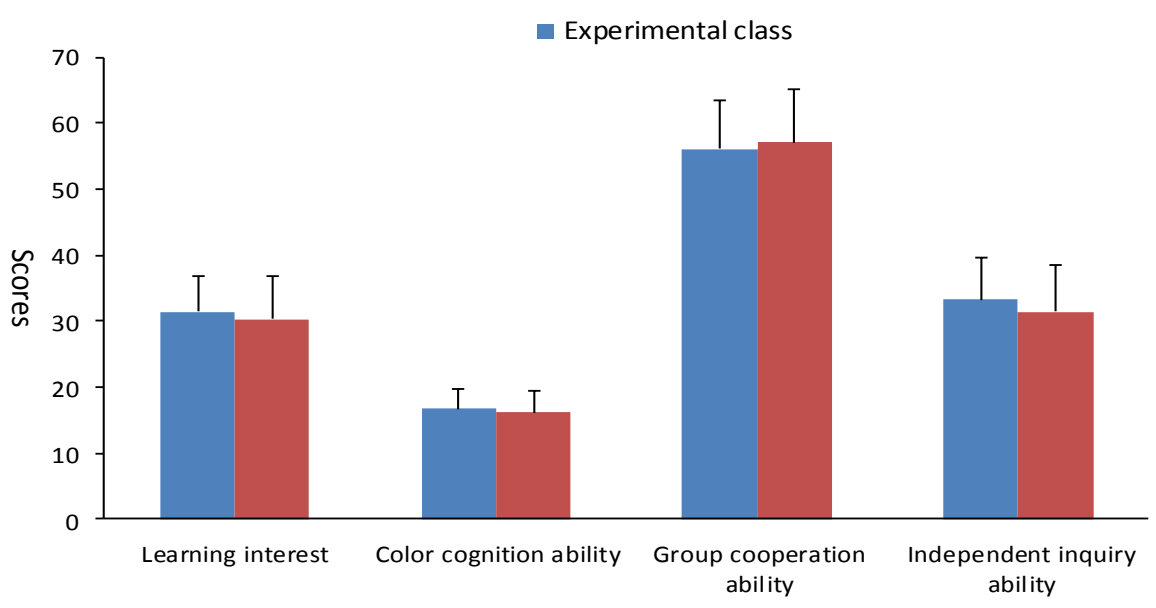

Figure 6. Comparison of learning indexes of both classes before teaching experiment

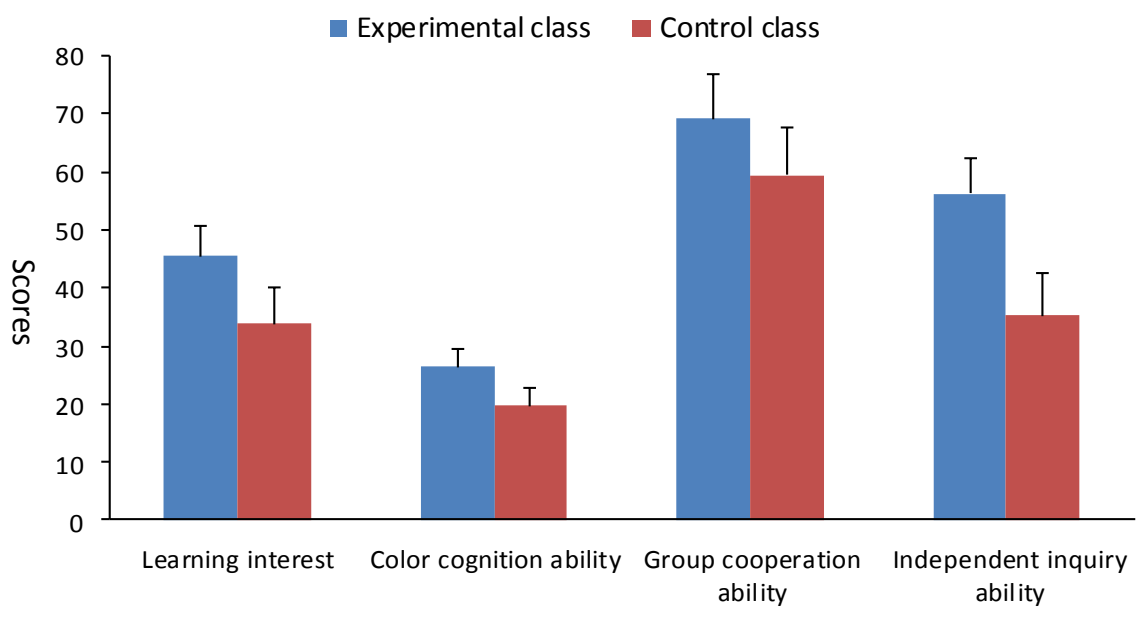

Figure 7. Comparison of learning indexes of both classes after teaching experiment 
The final examination average scores of the experimental class and control class show significant differences. In addition, communication with the students in experimental class reveals that more than $80 \%$ of students express projection color auto-adjustment multimedia system, has good intuitive property, and can show a more vivid and clearer color of landscape architecture design drawing and material object diagram. With this system, the teaching process designed by the teacher is also very interesting. Therefore, the system greatly helps in stimulating the students' learning interest.

Landscape Design is a required course of landscape architecture specialty. In the previous teaching mode, the teacher gets used to applying traditional multimedia equipment as the carrier to organize teaching practice. The exanimation of this course is based on test paper examination and supplemented by practical training examination. The whole teaching process lacks innovation. Thus, stimulating the students' learning initiative is challenging. Meanwhile, the whole teaching process cannot play a role in reforming course teaching. The present paper developed and designed a projection color auto-adjustment multimedia system. This system consists of a mobile device, projection equipment, and projection background, including multiple modules. Artificial intelligence adaptive module in the system can carry out adaptive adjustment of the color system gathered by projection background so that the pictures projected on the large screen are clearer with a more vivid color effect. Therefore, the system improves the teaching quality of Landscape Design, including display, appreciation, communication, and examination. Second, with the multimedia system, the classroom interaction mode between the teacher and students becomes more diversified. The learning process makes the teacher and the students pleased and relaxed, which naturally plays a positive role in improving examination scores.

\section{CONCLUSION}

The projection color auto-adjustment multimedia system is a new intelligent and information-based projection device that is different from previous multimedia display equipment. The striking feature of this system is that the artificial intelligence self-adaption module can achieve self-adaption of projection background color so that the projection background can reflect the material object's original appearance, projection color quality, and definition in the best way. Teaching experiment contrast results prove that compared with traditional multimedia equipment teaching, this system has remarkable value in terms of improving the teaching quality of Landscape Design. Therefore, this system deserves further promotion in other courses of landscape architecture design specialty.

\section{REFERENCES}

[1] Alkhabbaz Y., Tsutou A.T., Honjo M., "The effect of concurrent image projection technique on gait training for post total knee arthroplasty patients," Physiotherapy, vol. 101, no. S1, pp. e54-e55, May 2015. http://dx.doi.org/10.1016/j.physio.2015.03.173

[2] Minkel J.L., "Integration of Complex Rehabilitation Technology (CRT) into Long-term Care Supports," Topics in Geriatric Rehabilitation, vol. 31, no. 1, pp. 3-9, March 2015. http://dx.doi.org/10.1097/TGR.0000000000000044

[3] Klimberg R.K., Lawrence K.D., "Applying technology forecasting to new product development target setting of LCD panels," Emerald, no. 9, pp. 137-152, September 2013.

[4] Zheng G.T., Liu P.T., Wu M.C., et al., "Design of Bidirectional and Low Power Consumption Gate Driver in Amorphous Silicon, Technology for TFT-LCD Application," Journal of Display Technology, vol. 9, no. 2, pp. 91-99, February 2013. http://dx.doi.org/10.1109/JDT.2012.2225406

[5] Smith R., Day A., Rockall T., et al., "Advanced stereoscopic projection technology significantly improves novice performance of minimally invasive surgical skills," Surgical endoscopy, vol. 26 no. 6, pp. 1522-1527, January 2012. http://dx.doi.org/10.1007/ s00464-011-2080-8

[6] Stone J.A., Panchuk D., Davids K., et al., "Integrating advanced visual information with ball projection technology constrains dynamic interceptive actions," Procedia Engineering, vol. 72, pp. 156-161, December 2014. http://dx.doi.org/10.1016/j.proeng. 2014.06.027

[7] Shu S., Hu Q., "Analysis on application of computer and multimedia projection technology in college psychological experiment teaching," Business Conditions, vol. 39, pp. 263-263, September 2014.

[8] Tao T.T., He J.J., Zhang Y.P., "Application of immersion-type virtual environment of projective plane in teaching system," The Chinese Journal of ICT in Education Basic Education, no. 1, pp. 70-72, January 2014.

[9] Liu Y., Liu D., Huang Z.Z., et al., "Application of microscopic projection system in teaching "Foundation of Clinical Examination'," Laboratory Science, vol. 18, no. 2, pp. 112-114, April 2015 .

\section{AUTHOR}

Yue Wang is an Assistant Teacher at Sichuan University of Science and Engingeering, School of Fine Arts, Zigong, Sichuan, China. His research interests include Color Auto-adjustment Technology and Landscape Design. (15881309287@163.com)

This work was supported by Sichuan landscape architecture art and Design Engineering Research Center Project (2015YB03), Teaching reform project of Sichuan University of Science and Engineering (JG156). The authors declare that there is no conflict of interests. Submitted 07 August 2016. Published as resubmitted by the authors 14 September 2016. 